\title{
On positive solutions of $p$-Laplacian-type equations
}

\author{
Yehuda Pinchover \\ Department of Mathematics \\ Technion - Israel Institute of Technology \\ Haifa 32000, Israel \\ pincho@techunix.technion.ac.il
}

\author{
Kyril Tintarev \\ Department of Mathematics \\ Uppsala University \\ SE-751 06 Uppsala, Sweden \\ kyril.tintarev@math.uu.se
}

November 6, 2018

Dedicated to Vladimir Maz'ya on the occasion of his 70th birthday

\begin{abstract}
Let $\Omega$ be a domain in $\mathbb{R}^{d}, d \geq 2$, and $1<p<\infty$. Fix $V \in L_{\text {loc }}^{\infty}(\Omega)$. Consider the functional $Q$ and its Gâteaux derivative $Q^{\prime}$ given by

$Q(u):=\frac{1}{p} \int_{\Omega}\left(|\nabla u|^{p}+V|u|^{p}\right) \mathrm{d} x, \quad Q^{\prime}(u):=-\nabla \cdot\left(|\nabla u|^{p-2} \nabla u\right)+V|u|^{p-2} u$.

In this paper we discuss several aspects of relations between functionalanalytic properties of the functional $Q$ and properties of positive solutions of the equation $Q^{\prime}(u)=0$.

2000 Mathematics Subject Classification. Primary 35J60; Secondary 35J20, 35J70, 49R50.

Keywords. quasilinear elliptic operator, $p$-Laplacian, ground state, positive solutions, comparison principle, minimal growth.
\end{abstract}

\section{Introduction and Preliminaries}

Properties of positive solutions of quasilinear elliptic equations, and in particular of equations with the $p$-Laplacian term in the principal part, have been 
extensively studied over the recent decades, (see for example [3, 4, 28, 32 ] and the references therein). Fix $p \in(1, \infty)$, a domain $\Omega \subseteq \mathbb{R}^{d}$ and a real valued potential $V \in L_{\mathrm{loc}}^{\infty}(\Omega)$. The $p$-Laplacian equation in $\Omega$ with potential $V$ is the equation of the form

$$
-\Delta_{p}(u)+V|u|^{p-2} u=0 \quad \text { in } \Omega,
$$

where $\Delta_{p}(u):=\nabla \cdot\left(|\nabla u|^{p-2} \nabla u\right)$ is the celebrated $p$-Laplacian. This equation, in the semistrong sense, is a critical point equation for the functional

$$
Q(u)=Q_{V}(u):=\frac{1}{p} \int_{\Omega}\left(|\nabla u|^{p}+V|u|^{p}\right) \mathrm{d} x \quad u \in C_{0}^{\infty}(\Omega) .
$$

So, we consider solutions of (1.1) in the following weak sense.

Definition 1.1. A function $v \in W_{\text {loc }}^{1, p}(\Omega)$ is a (weak) solution of the equation

$$
Q^{\prime}(u):=-\Delta_{p}(u)+V|u|^{p-2} u=0 \quad \text { in } \Omega,
$$

if for every $\varphi \in C_{0}^{\infty}(\Omega)$

$$
\int_{\Omega}\left(|\nabla v|^{p-2} \nabla v \cdot \nabla \varphi+V|v|^{p-2} v \varphi\right) \mathrm{d} x=0
$$

We say that a real function $v \in C_{\text {loc }}^{1}(\Omega)$ is a supersolution (resp. subsolution) of the equation (1.3) if for every nonnegative $\varphi \in C_{0}^{\infty}(\Omega)$

$$
\int_{\Omega}\left(|\nabla v|^{p-2} \nabla v \cdot \nabla \varphi+V|v|^{p-2} v \varphi\right) \mathrm{d} x \geq 0(\text { resp. } \leq 0) .
$$

Next, we mention local properties of solutions of (1.3) that hold in any smooth subdomain $\Omega^{\prime} \Subset \Omega$ (i.e., $\overline{\Omega^{\prime}}$ is compact in $\Omega$ ).

1. Smoothness and Harnack inequality. Weak solutions of (1.3) admit Hölder continuous first derivatives, and nonnegative solutions of (1.3) satisfy the Harnack inequality (see for example [14, 34, 35, 32, 38]).

2. Principal eigenvalue and eigenfunction. For any smooth subdomain $\Omega^{\prime} \Subset \Omega$ consider the variational problem

$$
\lambda_{1, p}\left(\Omega^{\prime}\right):=\inf _{u \in W_{0}^{1, p}\left(\Omega^{\prime}\right)} \frac{\int_{\Omega^{\prime}}\left(|\nabla u|^{p}+V|u|^{p}\right) \mathrm{d} x}{\int_{\Omega^{\prime}}|u|^{p} \mathrm{~d} x} .
$$


It is well-known that for such a subdomain, (1.6) admits (up to a multiplicative constant) a unique minimizer $\varphi[9,13]$. Moreover, $\varphi$ is a positive solution of the quasilinear eigenvalue problem

$$
\begin{cases}Q^{\prime}(\varphi)=\lambda_{1, p}\left(\Omega^{\prime}\right)|\varphi|^{p-2} \varphi & \text { in } \Omega^{\prime} \\ \varphi=0 & \text { on } \partial \Omega^{\prime}\end{cases}
$$

$\lambda_{1, p}\left(\Omega^{\prime}\right)$ and $\varphi$ are called, respectively, the principal eigenvalue and eigenfunction of the operator $Q^{\prime}$ in $\Omega^{\prime}$.

\section{Weak and strong maximum principles.}

Theorem $1.2\left(\left[13\right.\right.$ (see also [3, 4])). Assume that $\Omega \subset \mathbb{R}^{d}$ is a bounded $C^{1+\alpha}$-domain, where $0<\alpha \leq 1$. Consider a functional $Q$ of the form (1.2) with $V \in L^{\infty}(\Omega)$. The following assertions are equivalent:

(i) $Q^{\prime}$ satisfies the maximum principle: If $u$ is a solution of the equation $Q^{\prime}(u)=f \geq 0$ in $\Omega$ with some $f \in L^{\infty}(\Omega)$, and satisfies $u \geq 0$ on $\partial \Omega$, then $u$ is nonnegative in $\Omega$.

(ii) $Q^{\prime}$ satisfies the strong maximum principle: If $u$ is a solution of the equation $Q^{\prime}(u)=f \supsetneqq 0$ in $\Omega$ with some $f \in L^{\infty}(\Omega)$, and satisfies $u \geq 0$ on $\partial \Omega$, then $u>0$ in $\Omega$.

(iii) $\lambda_{1, p}(\Omega)>0$.

(iv) For some $0 \supsetneqq f \in L^{\infty}(\Omega)$ there exists a positive strict supersolution $v$ satisfying $Q^{\prime}(v)=f$ in $\Omega$, and $v=0$ on $\partial \Omega$.

(iv') There exists a positive strict supersolution $v \in W^{1, p}(\Omega) \cap L^{\infty}(\Omega)$ satisfying $Q^{\prime}(v)=f \supsetneqq 0$ in $\Omega$, such that $\left.v\right|_{\partial \Omega} \in C^{1+\alpha}(\partial \Omega)$ and $f \in L^{\infty}(\Omega)$.

(v) For each nonnegative $f \in C^{\alpha}(\Omega) \cap L^{\infty}(\Omega)$ there exists a unique weak nonnegative solution of the problem $Q^{\prime}(u)=f$ in $\Omega$, and $u=0$ on $\partial \Omega$.

4. Weak comparison principle. We recall also the following weak comparison principle (or WCP for brevity).

Theorem 1.3 ([13]). Let $\Omega \subset \mathbb{R}^{d}$ be a bounded domain of class $C^{1, \alpha}$, where $0<\alpha \leq 1$, and suppose that $V \in L^{\infty}(\Omega)$. Assume that $\lambda_{1, p}(\Omega)>0$ and let 
$u_{i} \in W^{1, p}(\Omega) \cap L^{\infty}(\Omega)$ satisfying $Q^{\prime}\left(u_{i}\right) \in L^{\infty}(\Omega),\left.u_{i}\right|_{\partial \Omega} \in C^{1+\alpha}(\partial \Omega)$, where $i=1,2$. Suppose further that the following inequalities are satisfied

$$
\left\{\begin{aligned}
Q^{\prime}\left(u_{1}\right) & \leq Q^{\prime}\left(u_{2}\right) & & \text { in } \Omega \\
Q^{\prime}\left(u_{2}\right) & \geq 0 & & \text { in } \Omega \\
u_{1} & \leq u_{2} & & \text { on } \partial \Omega \\
u_{2} & \geq 0 & & \text { on } \partial \Omega
\end{aligned}\right.
$$

Then

$$
u_{1} \leq u_{2} \quad \text { in } \Omega
$$

\section{Strong comparison principle.}

Definition 1.4. we say that the strong comparison principle (or SCP for brevity) holds true for the functional $Q$ if the conditions of Theorem 1.3 implies that $u_{1}<u_{2}$ in $\Omega$ unless $u_{1}=u_{2}$ in $\Omega$.

Remark 1.5. It is well known that the SCP holds true for $p=2$ and for $p$-harmonic functions. For sufficient conditions for the validity of the SCP see [2, 5, 7, 16, 32, 37] and the references therein. In [5] M. Cuesta and P. Takáč present a counterexample where the WCP holds true but the SCP does not.

Throughout this paper we assume that

$$
Q(u) \geq 0 \quad \forall u \in C_{0}^{\infty}(\Omega) .
$$

The following Allegretto-Piepenbrink-type theorem, links the existence of positive solutions with the positivity of $Q$.

Theorem 1.6 ([28, Theorem 2.3]). Consider a functional $Q$ of the form (1.2). The following assertions are equivalent:

(i) The functional $Q$ is nonnegative on $C_{0}^{\infty}(\Omega)$.

(ii) Equation (1.3) admits a global positive solution.

(iii) Equation (1.3) admits a global positive supersolution.

In this paper we survey further connections between functional-analytic properties of the functional $Q$ and properties of its positive solutions. In particular, we review the following topics: 
- A representation of the nonnegative functional $Q$ as an integral of a nonnegative Lagrangian density and the existence of a useful equivalent nonnegative Lagrangian density with a simplified form (Section 22).

- The equivalence of several weak coercivity properties of $Q$. The characterization of the non-coercive case in terms of a Poincaré-type inequality, in terms of the existence of a generalized ground state, and in terms of the variational capacity of balls (Section 3).

- The identification of ground state as a global minimal solution (Section (4).

- A theorem of Liouville type connecting the behavior of a ground state of one functional with the existence of a ground state of another functional with a given 'decaying' subsolution (Section 5).

- A variational principle that characterizes solutions of minimal growth at infinity (Section 6).

- The existence of solutions to the inhomogeneous equation $Q^{\prime}(u)=f$ in the absence of the ground state (Section 7).

- The dependence of weak coercivity on the potential and the domain (Section 8). In particular, in Theorem 8.6, we extend the result for $p=2$ proved in [30, Theorem 2.9].

- Properties verified only in the linear case $(p=2)$, in particular, the definition of a natural functional space associated with the functional $Q$ (Section 9).

\section{Positive Lagrangian representations}

Let $v \in C_{\mathrm{loc}}^{1}(\Omega)$ be a positive solution (resp. subsolution) of (1.3). Using the positive Lagrangian representation from [3, 4, 6], we infer that for every $u \in C_{0}^{\infty}(\Omega), u \geq 0$,

$$
Q(u)=\int_{\Omega} L(u, v) \mathrm{d} x, \quad \text { resp. } Q(u) \leq \int_{\Omega} L(u, v) \mathrm{d} x,
$$


where

$$
L(u, v):=\frac{1}{p}\left[|\nabla u|^{p}+(p-1) \frac{u^{p}}{v^{p}}|\nabla v|^{p}-p \frac{u^{p-1}}{v^{p-1}} \nabla u \cdot|\nabla v|^{p-2} \nabla v\right] .
$$

It can be easily verified that $L(u, v) \geq 0$ in $\Omega[3,4]$.

Let now $w:=u / v$, where $v$ is a positive solution of (1.3) and $u \in C_{0}^{\infty}(\Omega)$, $u \geq 0$. Then (2.1) implies that

$$
Q(v w)=\frac{1}{p} \int_{\Omega}\left[|w \nabla v+v \nabla w|^{p}-w^{p}|\nabla v|^{p}-p w^{p-1} v|\nabla v|^{p-2} \nabla v \cdot \nabla w\right] \mathrm{d} x .
$$

Similarly, if $v$ is a nonnegative subsolution of (1.3), then

$$
Q(v w) \leq \frac{1}{p} \int_{\Omega}\left[|w \nabla v+v \nabla w|^{p}-w^{p}|\nabla v|^{p}-p w^{p-1} v|\nabla v|^{p-2} \nabla v \cdot \nabla w\right] \mathrm{d} x .
$$

Therefore, a nonnegative functional $Q$ can be represented as the integral of a nonnegative Lagrangian $L$. In spite of the nonnegativity of the in (2.1) and (2.3), the expression (2.2) of $L$ contains an indefinite term which poses obvious difficulties for extending the domain of the functional to more general weakly differentiable functions. The next proposition shows that $Q$ admits a two-sided estimate by a simplified Lagrangian containing only nonnegative terms. We call the functional associated with this simplified Lagrangian the simplified energy.

Let $f$ and $g$ be two nonnegative functions. We denote $f \asymp g$ if there exists a positive constant $C$ such that $C^{-1} g \leq f \leq C g$.

Proposition 2.1 ([26, Lemma 2.2]). Let $v \in C_{\mathrm{loc}}^{1}(\Omega)$ be a positive solution of (1.3). Then

$$
Q(v w) \asymp \int_{\Omega} v^{2}|\nabla w|^{2}(w|\nabla v|+v|\nabla w|)^{p-2} \mathrm{~d} x \quad \forall w \in C_{0}^{1}(\Omega), w \geq 0 .
$$

In particular, for $p \geq 2$, we have

$$
Q(v w) \asymp \int_{\Omega}\left(v^{p}|\nabla w|^{p}+v^{2}|\nabla v|^{p-2} w^{p-2}|\nabla w|^{2}\right) \mathrm{d} x \quad \forall w \in C_{0}^{1}(\Omega), w \geq 0 .
$$

If $v$ is only a nonnegative subsolution of (1.3), then for $1<p<\infty$ we have

$$
Q(v w) \leq C \int_{\Omega \cap\{v>0\}} v^{2}|\nabla w|^{2}(w|\nabla v|+v|\nabla w|)^{p-2} \mathrm{~d} x \quad \forall w \in C_{0}^{1}(\Omega), w \geq 0 .
$$


In particular, for $p \geq 2$ we have

$$
Q(v w) \leq C \int_{\Omega}\left(v^{p}|\nabla w|^{p}+v^{2}|\nabla v|^{p-2} w^{p-2}|\nabla w|^{2}\right) \mathrm{d} x \quad \forall w \in C_{0}^{1}(\Omega), w \geq 0 .
$$

Remark 2.2. It is shown in [26] that for $p>2$ neither of the terms in the simplified energy (2.6) is dominated by the other, so that (2.6) cannot be further simplified.

\section{Coercivity and ground state}

It is well known (see [21]) that for a nonnegative Schrödinger operator $P$ we have the following dichotomy: either there exists a strictly positive potential $W$ such that the Schrödinger operator $P-W$ is nonnegative, or $P$ admits a unique (generalized) ground state. It turns out that this statement is also true for nonnegative functionals of the form (1.2).

Definition 3.1. Let $Q$ be a nonnegative functional on $C_{0}^{\infty}(\Omega)$ of the form (1.2). We say that a sequence $\left\{u_{k}\right\} \subset C_{0}^{\infty}(\Omega)$ of nonnegative functions is a null sequence of the functional $Q$ in $\Omega$, if there exists an open set $B \Subset \Omega$ such that $\int_{B}\left|u_{k}\right|^{p} \mathrm{~d} x=1$, and

$$
\lim _{k \rightarrow \infty} Q\left(u_{k}\right)=\lim _{k \rightarrow \infty} \int_{\Omega}\left(\left|\nabla u_{k}\right|^{p}+V\left|u_{k}\right|^{p}\right) \mathrm{d} x=0
$$

We say that a positive function $v \in C_{\text {loc }}^{1}(\Omega)$ is a ground state of the functional $Q$ in $\Omega$ if $v$ is an $L_{\text {loc }}^{p}(\Omega)$ limit of a null sequence of $Q$. If $Q \geq 0$, and $Q$ admits a ground state in $\Omega$, we say that $Q$ is critical in $\Omega$.

Remark 3.2. The requirement that $\left\{u_{k}\right\} \subset C_{0}^{\infty}(\Omega)$, can clearly be weakened by assuming only that $\left\{u_{k}\right\} \subset W_{0}^{1, p}(\Omega)$. Also, as it follows from Theorem 3.4, the requirement $\int_{B}\left|u_{k}\right|^{p} \mathrm{~d} x=1$ can be replaced by $\int_{B}\left|u_{k}\right|^{p} \mathrm{~d} x \asymp 1$ or by $\int_{B} u_{k} \mathrm{~d} x \asymp 1$.

The following statements are based on rephrased statements of [28, Theorem 1.6], [29, Theorem 4.3] and [36, Proposition 3.1] (cf. [21, 27] for the case $p=2$ ).

Theorem 3.3. Suppose that the functional $Q$ is nonnegative on $C_{0}^{\infty}(\Omega)$. 
1. Any ground state $v$ is a positive solution of (1.3).

2. $Q$ admits a ground state $v$ if and only if (1.3) admits a unique positive supersolution.

3. $Q$ is critical in $\Omega$ if and only if $Q$ admits a null sequence that converges locally uniformly in $\Omega$.

4. If $Q$ admits a ground state $v$, then the following Poincaré type inequality holds: There exists a positive continuous function $W$ in $\Omega$, such that for every $\psi \in C_{0}^{\infty}(\Omega)$ satisfying $\int \psi v \mathrm{~d} x \neq 0$ there exists a constant $C>0$ such that the following inequality holds:

$$
Q(u)+C\left|\int_{\Omega} \psi u \mathrm{~d} x\right|^{p} \geq C^{-1} \int_{\Omega} W\left(|\nabla u|^{p}+|u|^{p}\right) \mathrm{d} x \quad \forall u \in C_{0}^{\infty}(\Omega) .
$$

The following theorem slightly extends [28, Theorem 1.6] in the spirit of [36, Proposition 3.1].

Theorem 3.4. Suppose that the functional $Q$ is nonnegative on $C_{0}^{\infty}(\Omega)$. The following statements are equivalent.

(a) $Q$ does not admit a ground state in $\Omega$.

(b) There exists a continuous function $W>0$ in $\Omega$ such that

$$
Q(u) \geq \int_{\Omega} W(x)|u(x)|^{p} \mathrm{~d} x \quad \forall u \in C_{0}^{\infty}(\Omega) .
$$

(c) There exists a continuous function $W>0$ in $\Omega$ such that

$$
Q(u) \geq \int_{\Omega} W(x)\left(|\nabla u(x)|^{p}+|u(x)|^{p}\right) \mathrm{d} x \quad \forall u \in C_{0}^{\infty}(\Omega) .
$$

(d) There exists an open set $B \Subset \Omega$ and $C_{B}>0$ such that

$$
Q(u) \geq C_{B}\left|\int_{B} u(x) \mathrm{d} x\right|^{p} \quad \forall u \in C_{0}^{\infty}(\Omega) .
$$


Suppose further that $d>p$. Then $Q$ does not admit a ground state in $\Omega$ if and only if there exists a continuous function $W>0$ in $\Omega$ such that

$$
Q(u) \geq\left(\int_{\Omega} W(x)|u(x)|^{p^{*}} \mathrm{~d} x\right)^{p / p^{*}} \quad \forall u \in C_{0}^{\infty}(\Omega),
$$

where $p^{*}=p d /(d-p)$ is the critical Sobolev exponent.

Definition 3.5. A nonnegative functional $Q$ on $C_{0}^{\infty}(\Omega)$ of the form (1.2) which is not critical is said to be subcritical (or weakly coercive) in $\Omega$.

Example 3.6. Consider the functional $Q(u):=\int_{\mathbb{R}^{d}}|\nabla u|^{p} \mathrm{~d} x$. It follows from [20, Theorem 2] that if $d \leq p$, then $Q$ admits a ground state $\varphi=$ constant in $\mathbb{R}^{d}$. On the other hand, if $d>p$, then

$$
u(x):=\left[1+|x|^{p /(p-1)}\right]^{(p-d) / p}, \quad v(x):=\text { constant }
$$

are two positive supersolutions of the equation $-\Delta_{p} u=0$ in $\mathbb{R}^{d}$. Therefore, $Q$ is weakly coercive in $\mathbb{R}^{d}$.

Example 3.7. Let $d>1, d \neq p$, and $\Omega:=\mathbb{R}^{d} \backslash\{0\}$ be the punctured space. The following celebrated Hardy's inequality holds true:

$$
Q_{\lambda}(u):=\int_{\Omega}\left(|\nabla u|^{p}-\lambda \frac{|u|^{p}}{|x|^{p}}\right) \mathrm{d} x \geq 0 \quad u \in C_{0}^{\infty}(\Omega),
$$

whenever $\lambda \leq c_{p, d}^{*}:=|(p-d) / p|^{p}$. Clearly, if $\lambda<c_{p, d}^{*}$, then $Q_{\lambda}(u)$ is weakly coercive. On the other hand, the proof of Theorem 1.3 in [31] shows that $Q_{\lambda}$ with $\lambda=c_{p, d}^{*}$ admits a null sequence. It can be easily checked that the function $v(r):=|r|^{(p-d) / p}$ is a positive solution of the corresponding radial equation:

$$
-\left|v^{\prime}\right|^{p-2}\left[(p-1) v^{\prime \prime}+\frac{d-1}{r} v^{\prime}\right]-c_{p, d}^{*} \frac{|v|^{p-2} v}{r^{p}}=0 \quad r \in(0, \infty) .
$$

Therefore, $\varphi(x):=|x|^{(p-d) / p}$ is the ground state of the equation

$$
-\Delta_{p} u-c_{p, d}^{*} \frac{|u|^{p-2} u}{|x|^{p}}=0 \quad \text { in } \Omega .
$$

Note that $\varphi \notin W_{\text {loc }}^{1, p}\left(\mathbb{R}^{d}\right)$ for $p \neq d$. In particular, $\varphi$ is not a positive supersolution of the equation $\Delta_{p} u=0$ in $\mathbb{R}^{d}$. 
In [39, 40] Troyanov has established a relationship between the $p$-capacity of closed balls in a Riemannian manifold $\mathcal{M}$ and the $p$-parabolicity of $\mathcal{M}$ with respect the $p$-Laplacian. We extend his definition and result to our case.

Definition 3.8. Suppose that the functional $Q$ is nonnegative on $C_{0}^{\infty}(\Omega)$. Let $K \Subset \Omega$ be a compact set. The $Q$-capacity of $K$ in $\Omega$ is defined by

$$
\operatorname{Cap}_{Q}(K, \Omega):=\inf \left\{Q(u) \mid u \in C_{0}^{\infty}(\Omega), u \geq 1 \text { on } K\right\} \text {. }
$$

Corollary 3.9. Suppose that the functional $Q$ is nonnegative on $C_{0}^{\infty}(\Omega)$. Then $Q$ is critical in $\Omega$ if and only if the $Q$-capacity of each closed ball in $\Omega$ is zero.

\section{Ground states and minimal growth at in- finity}

Definition 4.1. Let $K_{0}$ be a compact set in $\Omega$. A positive solution $u$ of the equation $Q^{\prime}(u)=0$ in $\Omega \backslash K_{0}$ is said to be a positive solution of minimal growth in a neighborhood of infinity in $\Omega$ (or $u \in \mathcal{M}_{\Omega, K_{0}}$ for brevity) if for any compact set $K$ in $\Omega$, with a smooth boundary, such that $K_{0} \Subset \operatorname{int}(K)$, and any positive supersolution $v \in C((\Omega \backslash K) \cup \partial K)$ of the equation $Q^{\prime}(u)=0$ in $\Omega \backslash K$, the inequality $u \leq v$ on $\partial K$ implies that $u \leq v$ in $\Omega \backslash K$.

A (global) positive solution $u$ of the equation $Q^{\prime}(u)=0$ in $\Omega$, which has minimal growth in a neighborhood of infinity in $\Omega$ (i.e. $u \in \mathcal{M}_{\Omega, \emptyset}$ ) is called a global minimal solution of the equation $Q^{\prime}(u)=0$ in $\Omega$.

Theorem 4.2 ([29, Theorem 5.1], cf. [28]). Suppose that $1<p<\infty$, and $Q$ is nonnegative on $C_{0}^{\infty}(\Omega)$. Then for any $x_{0} \in \Omega$ the equation $Q^{\prime}(u)=0$ has a positive solution $u \in \mathcal{M}_{\Omega,\left\{x_{0}\right\}}$.

We have the following connection between the existence of a global minimal solutions and weak coercivity.

Theorem 4.3 ([29, Theorem 5.2], cf. [28]). Let $1<p<\infty$, and assume that $Q$ is nonnegative on $C_{0}^{\infty}(\Omega)$. Then $Q$ is subcritical in $\Omega$ if and only if the equation $Q^{\prime}(u)=0$ in $\Omega$ does not admit a global minimal solution of the equation $Q^{\prime}(u)=0$ in $\Omega$. In particular, $u$ is ground state of the equation $Q^{\prime}(u)=0$ in $\Omega$ if and only $u$ is a global minimal solution of this equation. 
Consider a positive solution $u$ of the equation $Q^{\prime}(u)=0$ in a punctured neighborhood of $x_{0}$ which has a nonremovable singularity at $x_{0} \in \mathbb{R}^{d}$. Without loss of generality we may assume that $x_{0}=0$. If $1<p \leq d$, then the behavior of $u$ near an isolated singularity is well understood. Indeed, due to a result of L. Véron (see [28, Lemma 5.1]), we have that

$$
u(x) \sim\left\{\begin{array}{ll}
|x|^{\alpha(d, p)} & p<d, \\
-\log |x| & p=d,
\end{array} \quad \text { as } x \rightarrow 0,\right.
$$

where $\alpha(d, p):=(p-d) /(p-1)$, and $f \sim g$ means that

$$
\lim _{x \rightarrow 0} \frac{f(x)}{g(x)}=C
$$

for some positive constant $C$. In particular, $\lim _{x \rightarrow 0} u(x)=\infty$.

Assume now that $p>d$. A general question is whether in this case, any positive solution of the equation $Q^{\prime}(u)=0$ in a punctured ball centered at $x_{0}$ can be continuously extended at $x_{0}$ (see [17] for partial results).

Under the assumption that $u \asymp 1$ near the isolated point the answer is given by Lemma 5.3 in [29]:

Lemma 4.4. Assume that $p>d$, and let $v$ be a positive solution of the equation $Q^{\prime}(u)=0$ in a punctured neighborhood of $x_{0}$ satisfying $u \asymp 1$ near $x_{0}$. Then $u$ can be continuously extended at $x_{0}$.

The following statement combines the second part of [28, Theorem 5.4], where the case $1<p \leq d$ is considered with [29, Theorem 5.3] which deals with the case $p>d$.

Theorem 4.5. Let $x_{0} \in \Omega$, and let $u \in \mathcal{M}_{\Omega,\left\{x_{0}\right\}}$. Then $Q$ is subcritical in $\Omega$ if and only if $u$ has a nonremovable singularity at $x_{0}$.

\section{Liouville theorems}

In [26] we use some of the positivity properties of the nonnegative functional $Q$ discussed in the previous sections to prove a Liouville comparison principle for equations $Q^{\prime}(u)=0$ in $\Omega$. (see Theorem 9.1 for the case $p=2$ ). 
Theorem 5.1. Let $\Omega$ be a domain in $\mathbb{R}^{d}, d \geq 1$, and let $p \in(1, \infty)$. For $j=0,1$, let $V_{j} \in L_{\mathrm{loc}}^{\infty}(\Omega)$, and let

$$
Q_{j}(u):=\int_{\Omega}\left(|\nabla u(x)|^{p}+V_{j}(x)|u(x)|^{p}\right) \mathrm{d} x \quad u \in C_{0}^{\infty}(\Omega) .
$$

Assume that the following assumptions hold true.

(i) The functional $Q_{1}$ admits a ground state $\varphi$ in $\Omega$.

(ii) $Q_{0} \geq 0$ on $C_{0}^{\infty}(\Omega)$, and the equation $Q_{0}^{\prime}(u)=0$ in $\Omega$ admits a subsolution $\psi \in W_{\text {loc }}^{1, p}(\Omega)$ satisfying $\psi_{+} \neq 0$, where $\psi_{+}(x):=\max \{0, \psi(x)\}$.

(iii) The following inequality holds in $\Omega$

$$
\psi_{+} \leq C \varphi,
$$

where $C>0$ is a positive constant.

(iv) The following inequality holds in $\Omega$

$$
\left|\nabla \psi_{+}\right|^{p-2} \leq C|\nabla \varphi|^{p-2},
$$

where $C>0$ is a positive constant.

Then the functional $Q_{0}$ admits a ground state in $\Omega$, and $\psi$ is the ground state. In particular, $\psi$ is (up to a multiplicative constant) the unique positive supersolution of the equation $Q_{0}^{\prime}(u)=0$ in $\Omega$.

Remark 5.2. Condition (5.2) is redundant for $p=2$. For $p \neq 2$ it is equivalent to the assumption that the following inequality holds in $\Omega$ :

$$
\begin{cases}\left|\nabla \psi_{+}\right| \leq C|\nabla \varphi| & \text { if } p>2, \\ \left|\nabla \psi_{+}\right| \geq C|\nabla \varphi| & \text { if } p<2\end{cases}
$$

where $C>0$ is a positive constant.

Remark 5.3. This theorem holds if, in addition to (5.1), one assumes instead of $\left|\nabla \psi_{+}\right|^{p-2} \leq C|\nabla \varphi|^{p-2}$ in $\Omega$ (see (5.2)), that the following inequality holds true in $\Omega$

$$
\psi_{+}^{2}\left|\nabla \psi_{+}\right|^{p-2} \leq C \varphi^{2}|\nabla \varphi|^{p-2},
$$

where $C>0$ is a positive constant. 
Remark 5.4. Suppose that $1<p<2$, and assume that the ground state $\varphi>0$ of the functional $Q_{1}$ is such that $w=\mathbf{1}$ is a ground state of the functional

$$
E_{1}^{\varphi}(w)=\int_{\Omega} \varphi^{p}|\nabla w|^{p} \mathrm{~d} x
$$

that is, there is a sequence $\left\{w_{k}\right\} \subset C_{0}^{\infty}(\Omega)$ of nonnegative functions satisfying $E_{1}^{\varphi}\left(w_{k}\right) \rightarrow 0$, and $\int_{B}\left|w_{k}\right|^{p}=1$ for a fixed $B \Subset \Omega$ (this implies that $w_{k} \rightarrow \mathbf{1}$ in $\left.L_{\mathrm{loc}}^{p}(\Omega)\right)$. In this case, the conclusion of Theorem 5.1 holds if there is a nonnegative subsolution $\psi_{+}$of $Q_{0}^{\prime}(u)=0$ satisfying (5.1) alone, without an assumption on the gradients (like (5.2) or (5.4)).

Remark 5.5. Condition (5.2) is essential when $p>2$, and presumably also when $p<2$. When $p>2, \Omega=\mathbb{R}^{d}$ and $V$ is radially symmetric, Proposition 4.2 in [26] shows that the simplified energy functional is not equivalent to either of its two terms that lead to conditions (5.1) and (5.2), respectively.

Example 5.6. Assume that $1 \leq d \leq p \leq 2, p>1, \Omega=\mathbb{R}^{d}$, and consider the functional $Q_{1}(u):=\int_{\mathbb{R}^{d}}|\nabla u|^{p} \mathrm{~d} x$. By Example 3.6, the functional $Q_{1}$ admits a ground state $\varphi=$ constant in $\mathbb{R}^{d}$.

Let $Q_{0}$ be a functional of the form (1.2) satisfying $Q_{0} \geq 0$ on $C_{0}^{\infty}\left(\mathbb{R}^{d}\right)$. Let $\psi \in W_{\text {loc }}^{1, p}\left(\mathbb{R}^{d}\right), \psi_{+} \neq 0$ be a subsolution of the equation $Q_{0}^{\prime}(u)=0$ in $\mathbb{R}^{d}$, such that $\psi_{+} \in L^{\infty}\left(\mathbb{R}^{d}\right)$. It follows from Theorem 5.1 that $\psi$ is the ground state of $Q_{0}$ in $\mathbb{R}^{d}$. In particular, $\psi$ is (up to a multiplicative constant) the unique positive supersolution and unique bounded solution of the equation $Q_{0}^{\prime}(u)=0$ in $\mathbb{R}^{d}$. Note that there is no assumption on the behavior of the potential $V_{0}$ at infinity. This result generalizes some striking Liouville theorems for Schrödinger operators on $\mathbb{R}^{d}$ that hold for $d=1,2$ and $p=2$ (see [24, theorems 1.4-1.6]).

Example 5.7. Let $1<p<\infty, d>1, d \neq p$, and $\Omega:=\mathbb{R}^{d} \backslash\{0\}$ be the punctured space. Let $Q_{0}$ be a functional of the form (1.2) satisfying $Q_{0} \geq 0$ on $C_{0}^{\infty}(\Omega)$. Let $\psi \in W_{\text {loc }}^{1, p}(\Omega), \psi_{+} \neq 0$ be a subsolution of the equation $Q_{0}^{\prime}(u)=0$ in $\Omega$, satisfying

$$
\psi_{+}(x) \leq C|x|^{(p-d) / p} \quad x \in \Omega .
$$

When $p>2$, we require in addition that the following inequality is satisfied

$$
\psi_{+}(x)^{2}\left|\nabla \psi_{+}(x)\right|^{p-2} \leq C|x|^{2-d} \quad x \in \Omega .
$$


It follows from Theorem 5.1, Remark 5.3, Remark 5.4 and Example 3.7 that $\psi$ is the ground state of $Q_{0}$ in $\Omega$. Let $\varphi$ be the ground state of the Hardy functional. The reason that (5.7) is stated only for $p>2$ hinges on the property that for $1<p<2$ the functional

$$
E_{1}^{\varphi}(w)=\int_{\Omega}|x|^{p-d}|\nabla w|^{p} \mathrm{~d} x
$$

admits a ground state $\mathbf{1}$, so Remark 5.4 applies.

Next, we present a family of functionals $Q_{0}$ for which the conditions of Example 5.7 are satisfied.

Example 5.8. Let $d \geq 2,1<p<d, \alpha \geq 0$, and $\Omega:=\mathbb{R}^{d} \backslash\{0\}$. Let

$$
W_{\alpha}(x):=-\left(\frac{d-p}{p}\right)^{p} \frac{\alpha d p /(d-p)+|x|^{\frac{p}{p-1}}}{\left(\alpha+|x|^{\frac{p}{p-1}}\right)^{p}} .
$$

Note that if $\alpha=0$ this is the Hardy potential as in Example 3.7. If $Q_{0}$ is the functional (1.2) with the potential $V_{0}:=W_{\alpha}$, then

$$
\psi_{\alpha}(x):=\left(\alpha+|x|^{\frac{p}{p-1}}\right)^{-\frac{(d-p)(p-1)}{p^{2}}}
$$

is a solution of $Q_{0}^{\prime}(u)=0$ in $\Omega$, and therefore $Q_{0} \geq 0$ on $C_{0}^{\infty}(\Omega)$. Moreover, one can use Example 5.7 to show that $\psi_{\alpha}$ is a ground state of $Q_{0}$. Note first that $\psi=\psi_{\alpha}$ satisfies (5.6). If $\frac{d}{d-1}<p<d$, then $\psi_{\alpha}$ satisfies also (5.7) and therefore, it is a ground state in this case. In the remaining case $p \leq \frac{d}{d-1} \leq 2$, Example 3.7 concludes that $\psi_{\alpha}$ is a ground state from the property of the functional (5.8).

\section{Variational principle for solutions of mini- mal growth and comparison principle}

The aim of this section is to represent positive solutions of minimal growth in a neighborhood of infinity in $\Omega$ as a limit of a modified null sequence.

Theorem 6.1 ([29, Theorem 7.1]). Suppose that $1<p<\infty$, and let $Q_{V}$ be nonnegative on $C_{0}^{\infty}(\Omega)$. Let $\Omega_{1} \Subset \Omega$ be an open set, and let $u \in C\left(\Omega \backslash \Omega_{1}\right)$ be 
a positive solution of the equation $Q_{V}^{\prime}(u)=0$ in $\Omega \backslash \overline{\Omega_{1}}$ satisfying $|\nabla u| \neq 0$ in $\Omega \backslash \overline{\Omega_{1}}$.

Then $u \in \mathcal{M}_{\Omega, \overline{\Omega_{1}}}$ if for every smooth open set $\Omega_{2}$ satisfying $\Omega_{1} \Subset \Omega_{2} \Subset \Omega$, and an open set $B \Subset\left(\Omega \backslash \overline{\Omega_{2}}\right)$ there exists a sequence $\left\{u_{k}\right\} \subset C_{0}^{\infty}(\Omega), u_{k} \geq 0$, such that for all $k \in \mathbb{N}, \int_{B}\left|u_{k}\right|^{p} \mathrm{~d} x=1$, and

$$
\lim _{k \rightarrow \infty} \int_{\Omega \backslash \bar{\Omega}_{2}} L\left(u_{k}, u\right) \mathrm{d} x=0
$$

where $L$ is the Lagrangian given by (2.2).

Conjecture 6.2. We conjecture that for $p \neq 2$ a positive global solution of the equation $Q_{V}^{\prime}(u)=0$ in $\Omega$ satisfying $u \in \mathcal{M}_{\Omega, \overline{\Omega_{1}}}$ for some smooth open set $\Omega_{1} \Subset \Omega$ is a global minimal solution.

Remark 6.3. The validity of Conjecture 6.2 seems to be related to the SCP. We note that if Conjecture 6.2 holds true, then the condition of Theorem 6.1 is also necessary (cf. Section 9.3).

Finally, we formulate a sub-supersolution comparison principle for our singular elliptic equation.

Theorem 6.4 (Comparison Principle [29]). Assume that the functional $Q_{V}$ is nonnegative on $C_{0}^{\infty}(\Omega)$. Fix smooth open sets $\Omega_{1} \Subset \Omega_{2} \Subset \Omega$. Let $u, v \in$ $W_{\text {loc }}^{1, p}\left(\Omega \backslash \Omega_{1}\right) \cap C\left(\Omega \backslash \Omega_{1}\right)$ be, respectively, a positive subsolution and a supersolution of the equation $Q_{V}^{\prime}(w)=0$ in $\Omega \backslash \overline{\Omega_{1}}$ such that $u \leq v$ on $\partial \Omega_{2}$.

Assume further that $Q_{V}^{\prime}(u) \in L_{\mathrm{loc}}^{\infty}\left(\Omega \backslash \Omega_{1}\right),|\nabla u| \neq 0$ in $\Omega \backslash \overline{\Omega_{1}}$, and that there exist an open set $B \Subset\left(\Omega \backslash \overline{\Omega_{2}}\right)$ and a sequence $\left\{u_{k}\right\} \subset C_{0}^{\infty}(\Omega), u_{k} \geq 0$, such that

$$
\int_{B}\left|u_{k}\right|^{p} \mathrm{~d} x=1 \quad \forall k \geq 1, \text { and } \quad \lim _{k \rightarrow \infty} \int_{\Omega \backslash \bar{\Omega}_{1}} L\left(u_{k}, u\right) \mathrm{d} x=0 .
$$

Then $u \leq v$ on $\Omega \backslash \Omega_{2}$.

\section{Solvability of nonhomogeneous equation}

In this section we discuss some results of [36] concerning the solvability of the nonhomogeneous equation

$$
Q_{V}^{\prime}(u)=f \quad \text { in } \Omega,
$$


where $Q_{V}$ is the nonnegative functional (1.2). In some cases, e.g. $V \geq 0$ or $p=2$, the nonnegativity of $Q_{V}$ implies that $Q_{V}$ is convex. In general, however, $Q_{V}$ might be nonconvex. For $p>2$, see the elementary one-dimensional example at the end of [8], and also the proof of [13, Theorem 7]. For $p<2$, see Example 2 in [12].

If $Q_{V}$ is convex and weakly coercive, then (7.1) can be easily solved by defining a Banach space $X$ as a completion of $C_{0}^{\infty}(\Omega)$ with respect to the norm $Q_{V}(\cdot)^{1 / p}$ (see the discussion of the analogous space for $p=2$ in Section 9 below). Such space is continuously imbedded into $W_{\text {loc }}^{1, p}(\Omega)$ by (3.4). It follows that for every $f \in X^{*}$ the functional

$$
u \mapsto Q_{V}(u)-\langle f, u\rangle \quad u \in X,
$$

has a minimum that solves (7.1).

Note that the requirement of weak coercivity cannot be removed. Indeed, if $p=2, \Omega$ is smooth and bounded, and $V=0$, then the corresponding ground state $\varphi$ is the first eigenfunction of the Dirichlet Laplacian with an eigenvalue $\lambda_{0}$, and there is no solution $u \in W_{0}^{1,2}(\Omega)$ to the equation

$$
\left(-\Delta-\lambda_{0}\right) u=f \quad \text { in } \Omega
$$

unless $\int_{\Omega} \varphi(x) f(x) \mathrm{d} x=0$.

In order to address the nonconvex case, we use the following setup from convex analysis (see [10, Chapt. I] for details.) The polar (or conjugate) functional to $Q_{V}$ is defined by

$$
Q_{V}^{*}(f):=\sup _{u \in C_{0}^{\infty}(\Omega)}\left[\langle u, f\rangle-Q_{V}(u)\right] \quad f \in \mathcal{D}^{\prime}(\Omega) .
$$

Notice that $Q_{V}$ is positively homogeneous of degree $p$, and consequently, $Q_{V}^{*}$ is positively homogeneous functional of degree $p^{\prime}$, where $p^{\prime}=p /(p-1)$. The (effective or natural) domain $X^{*}$ of $Q_{V}^{*}$ is defined naturally by

$$
X^{*}=\left\{f \in \mathcal{D}^{\prime}(\Omega): Q_{V}^{*}(f)<\infty\right\} .
$$

The definition of $Q_{V}^{*}(f)$ in (17.3) yields immediately the well known FenchelYoung inequality

$$
|\langle u, f\rangle| \leq Q_{V}(u)+Q_{V}^{*}(f),
$$

and equivalently, the Hölder inequality

$$
|\langle u, f\rangle| \leq\left(p Q_{V}(u)\right)^{1 / p}\left(p^{\prime} Q_{V}^{*}(f)\right)^{1 / p^{\prime}} .
$$


One can easily verify that $X^{*}$ is a linear subspace of $\mathcal{D}^{\prime}(\Omega)$ and

$$
\|f\|_{*}:=\left(p^{\prime} Q_{V}^{*}(f)\right)^{1 / p^{\prime}}
$$

defines a norm on $X^{*}$. In particular, $\|f\|_{*}=0$ implies $f=0$, as a consequence of (7.6) combined with the separation property of the duality between $C_{0}^{\infty}(\Omega)$ and $\mathcal{D}^{\prime}(\Omega)$.

From (3.4) one immediately deduces that $X^{*}$ contains $\left(W_{0}^{1, p}(\Omega ; W)\right)^{\prime}$ and that the corresponding embedding

$$
\left(W_{0}^{1, p}(\Omega ; W)\right)^{\prime} \hookrightarrow X^{*}
$$

is continuous and dense. The density follows from

$$
C_{0}^{\infty}(\Omega) \subset\left(W_{0}^{1, p}(\Omega ; W)\right)^{\prime} \subset X^{*} \subset \mathcal{D}^{\prime}(\Omega) .
$$

Therefore, denoting by $X^{* *}$ the (strong) dual space of $X^{*}$ with respect to the duality $\langle\cdot, \cdot\rangle$, we observe that $X^{* *}$ is continuously embedded into $W^{1, p}(\Omega ; W)$ and that $C_{0}^{\infty}(\Omega)$ is weak-star dense in $X^{* *}$. It is noteworthy that the separability of $X^{*}$ in the norm topology implies that the weak-star topology on any bounded subset of $X^{* *}$ is metrizable (Rudin [33, Theorem 3.16 , p. 70]). Now consider the bipolar (or second conjugate) functional to $Q_{V}$ defined by

$$
Q_{V}^{* *}(u):=\sup _{f \in X^{*}}\left[\langle u, f\rangle-Q_{V}^{*}(f)\right] \quad u \in X^{* *} .
$$

From (17.5) it is evident that

$$
0 \leq Q_{V}^{* *}(u) \leq Q_{V}(u) \quad \text { for every } u \in C_{0}^{\infty}(\Omega) .
$$

Moreover, in analogy with the norm $\|\cdot\|_{*}$ on $X^{*}$ (see (7.7)), the dual norm $\|\cdot\|_{* *}$ on $X^{* *}$ is given by

$$
\|u\|_{* *}=\left(p Q_{V}^{* *}(u)\right)^{1 / p} .
$$

The Fenchel-Young and Hölder inequalities, (7.5) and (7.6), respectively, remain valid with $Q_{V}^{* *}(u)$ in place of $Q_{V}$. In particular, we have

$$
|\langle u, f\rangle| \leq\|u\|_{* *}\|f\|_{*} \leq\left(p Q_{V}(u)\right)^{1 / p}\|f\|_{*} \quad \text { for } f \in X^{*}, u \in C_{0}^{\infty}(\Omega) .
$$

It follows [10, Ch. I, §4], that for all $f \in X^{*}$,

$$
\inf _{u \in C_{0}^{\infty}(\Omega)}\left[Q_{V}(u)-\langle u, f\rangle\right]=\inf _{u \in C_{0}^{\infty}(\Omega)}\left[Q_{V}^{* *}(u)-\langle u, f\rangle\right]=-Q_{V}^{*}(f) .
$$


Definition 7.1. Given a distribution $f \in X^{*}$, we say that a function $u_{0} \in$ $W_{\text {loc }}^{1, p}(\Omega)$ is a generalized (or relaxed) minimizer for the functional $u \mapsto$ $Q_{V}(u)-\langle u, f\rangle$ if it has the following three properties:

(i) $\quad u_{0}$ is a (true) minimizer for the functional

$$
u \mapsto Q_{V}^{* *}(u)-\langle u, f\rangle: X^{* *} \rightarrow \mathbb{R},
$$

hence, $u_{0} \in X^{* *}$.

(ii) $u_{0}$ satisfies equation $Q_{V}^{\prime}(u)=f$ in the sense of distributions on $\Omega$.

(iii) There exists a (minimizing) sequence $\left\{u_{k}\right\}_{k=1}^{\infty} \subset C_{0}^{\infty}(\Omega)$ such that $Q_{V}\left(u_{k}\right)-\left\langle u_{k}, f\right\rangle \rightarrow-Q_{V}^{*}(f)$ and

$$
Q_{V}^{\prime}\left(u_{k}\right)-f \equiv Q_{V}^{\prime}\left(u_{k}\right)-\langle\cdot, f\rangle \rightarrow 0 \quad \text { strongly in } W_{\text {loc }}^{-1, p^{\prime}}(\Omega)
$$

as $k \rightarrow \infty$, together with $u_{k} \rightarrow u_{0}$ strongly in $W_{\text {loc }}^{1, p}(\Omega)$, and $u_{k} \stackrel{*}{\rightarrow} u_{0}$ weakly-star in $X^{* *}$ as $k \rightarrow \infty$.

We can now formulate the existence result.

Theorem 7.2 ([36, Theorem 4.3]). Let $\Omega \subset \mathbb{R}^{d}$ be a domain, $1<p<\infty$, and $V \in L_{\mathrm{loc}}^{\infty}(\Omega)$. Assume that the nonnegative functional $Q_{V}$ is weakly coercive. Then, for every $f \in X^{*}$, the functional $u \mapsto Q_{V}(u)-\langle u, f\rangle$ is bounded from below on $C_{0}^{\infty}(\Omega)$ and has a generalized minimizer $u_{0}$ in $X^{* *}\left(\subset W_{\mathrm{loc}}^{1, p}(\Omega)\right)$. In particular, this minimizer verifies the equation $Q_{V}^{\prime}(u)=f$.

\section{Criticality theory}

In this section we discuss positivity properties of the functional $Q$ from [28, 30] along the lines of criticality theory for second-order linear elliptic operators [22, 23]. We note that Theorem 8.6 and Example 8.7 are new results.

Proposition 8.1. Let $V_{i} \in L_{\mathrm{loc}}^{\infty}(\Omega)$. If $V_{2} \supsetneqq V_{1}$ and $Q_{V_{1}} \geq 0$, then $Q_{V_{2}}$ is subcritical (weakly coercive).

Proposition 8.2. Let $\Omega_{1} \subset \Omega_{2}$ be domains in $\mathbb{R}^{d}$ such that $\Omega_{2} \backslash \overline{\Omega_{1}} \neq \emptyset$. Let $Q_{V}$ be defined on $C_{0}^{\infty}\left(\Omega_{2}\right)$.

1. If $Q_{V} \geq 0$ on $C_{0}^{\infty}\left(\Omega_{2}\right)$, then $Q_{V}$ is subcritical in $\Omega_{1}$.

2. If $Q_{V}$ is critical in $\Omega_{1}$, then $Q_{V}$ is nonpositive in $\Omega_{2}$. 
Proposition 8.3. Let $V_{0}, V_{1} \in L_{\mathrm{loc}}^{\infty}(\Omega), V_{0} \neq V_{1}$. For $t \in \mathbb{R}$ we denote

$$
Q_{t}(u):=t Q_{V_{1}}(u)+(1-t) Q_{V_{0}}(u),
$$

and suppose that $Q_{V_{i}} \geq 0$ on $C_{0}^{\infty}(\Omega)$ for $i=0,1$.

Then $Q_{t} \geq 0$ on $C_{0}^{\infty}(\Omega)$ for all $t \in[0,1]$. Moreover, $Q_{t}$ is subcritical in $\Omega$ for all $t \in(0,1)$.

Proposition 8.4. Let $Q_{V}$ be a subcritical functional in $\Omega$. Consider $V_{0} \in$ $L^{\infty}(\Omega)$ such that $V_{0} \nsupseteq 0$ and $\operatorname{supp} V_{0} \Subset \Omega$. Then there exist $\tau_{+}>0$ and $-\infty \leq \tau_{-}<0$ such that $Q_{V+t V_{0}}$ is subcritical in $\Omega$ for $t \in\left(\tau_{-}, \tau_{+}\right)$, and $Q_{V+\tau_{+} V_{0}}$ is critical in $\Omega$.

Proposition 8.5. Assume that $Q_{V}$ admits a ground state $v$ in $\Omega$. Consider $V_{0} \in L^{\infty}(\Omega)$ such that $\operatorname{supp} V_{0} \Subset \Omega$. Then there exists $0<\tau_{+} \leq \infty$ such that $Q_{V+t V_{0}}$ is subcritical in $\Omega$ for $t \in\left(0, \tau_{+}\right)$if and only if

$$
\int_{\Omega} V_{0}|v|^{p} \mathrm{~d} x>0 .
$$

In propositions 8.4 and 8.5 we assumed that the perturbation $V_{0}$ has a compact support. In the following we consider a wider class of perturbations. Assume that $Q$ is subcritical in $\Omega$ and $d>p$. It follows from Theorem 3.4 that there exists a continuous weight function $W$ such that the following Hardy-Sobolev-Maz'ya inequality is satisfied

$$
Q(u) \geq\left(\int_{\Omega} W(x)|u(x)|^{p^{*}} \mathrm{~d} x\right)^{p / p^{*}} \quad \forall u \in C_{0}^{\infty}(\Omega),
$$

where $p^{*}=p d /(d-p)$ is the critical Sobolev exponent.

The following theorem shows that for a certain class of potentials $\tilde{V}$ the above Hardy-Sobolev-Maz'ya inequality is preserved with the same weight function $W$. This theorem extends the analogous result for $p=2$ proved in [30, Theorem 2.9]. We may say that such $\tilde{V}$ are small perturbations of the functional $Q$ in $\Omega$.

Theorem 8.6. Let $Q$ be the functional (1.2) with $d>p$ and suppose that

$$
Q(u) \geq\left(\int_{\Omega} W|u|^{p^{*}} \mathrm{~d} x\right)^{p / p^{*}} \quad u \in C_{0}^{\infty}(\Omega),
$$


where $W$ is some positive continuous function (see Theorem 3.4, and in particular (3.6)). Let

$$
\tilde{V} \in L_{\mathrm{loc}}^{\infty}(\Omega) \cap L^{d / p}\left(\Omega ; W^{-d / p^{*}}\right) .
$$

Consider the one-parameter family of functionals $\tilde{Q}_{\lambda}$ defined by

$$
\tilde{Q}_{\lambda}(u):=Q(u)+\lambda \int_{\Omega} \tilde{V}|u|^{p} \mathrm{~d} x \quad u \in C_{0}^{\infty}(\Omega),
$$

where $\lambda \in \mathbb{R}$, and let

$$
S:=\left\{\lambda \in \mathbb{R} \mid \tilde{Q}_{\lambda} \geq 0 \text { on } C_{0}^{\infty}(\Omega)\right\} .
$$

(i) If $\lambda \in S$ and $\tilde{Q}_{\lambda}$ is subcritical in $\Omega$, then there exists $C>0$ such that

$$
\tilde{Q}_{\lambda}(u) \geq C\left(\int_{\Omega} W|u|^{p^{*}} \mathrm{~d} x\right)^{p / p^{*}} \quad u \in C_{0}^{\infty}(\Omega) .
$$

(ii) If $\lambda \in S$ and $\tilde{Q}_{\lambda}$ admits a ground state $v$, then for every $\psi \in C_{0}^{\infty}(\Omega)$ such that $\int_{\Omega} \psi v \mathrm{~d} x \neq 0$ there exist $C, C_{1}>0$ such that the following HardySobolev-Maz'ya-Poincaré type inequality holds

$$
\tilde{Q}_{\lambda}(u)+C_{1}\left|\int_{\Omega} \psi u \mathrm{~d} x\right|^{p} \geq C\left(\int_{\Omega} W|u|^{p^{*}} \mathrm{~d} x\right)^{p / p^{*}} \quad u \in C_{0}^{\infty}(\Omega) .
$$

(iii) The set $S$ is a closed interval with a nonempty interior which is bounded if and only if $\tilde{V}$ changes its sign on a set of a positive measure in $\Omega$. Moreover, $\lambda \in \partial S$ if and only if $\tilde{Q}_{\lambda}$ is critical in $\Omega$.

Proof. (i)-(ii) Assume first that $\tilde{Q}_{\lambda}$ is subcritical in $\Omega$, and (8.6) does not hold, then there exists a sequence $\left\{u_{k}\right\} \subset C_{0}^{\infty}(\Omega)$ of nonnegative functions such that $\tilde{Q}_{\lambda}\left(u_{k}\right) \rightarrow 0$, and $\int_{\Omega} W\left|u_{k}\right|^{p^{*}} \mathrm{~d} x=1$. In light of (3.4) (with another weight function $\tilde{W})$ it follows that that $u_{k} \rightarrow 0$ in $W_{\text {loc }}^{1, p}(\Omega)$.

If $\tilde{Q}_{\lambda}$ has a ground state $v$, and (8.7) does not hold. Then there exists a sequence $\left\{u_{k}\right\} \subset C_{0}^{\infty}(\Omega)$ of nonnegative functions such that $\tilde{Q}_{\lambda}\left(u_{k}\right) \rightarrow 0$, $\int_{\Omega} \psi u_{k} \mathrm{~d} x \rightarrow 0$, but $\int_{\Omega} W\left|u_{k}\right|^{p^{*}} \mathrm{~d} x=1$. It follows from (3.2) (with another weight function $\tilde{W})$ that $u_{k} \rightarrow 0$ in $W_{\text {loc }}^{1, p}(\Omega)$.

Consequently, for any $K \Subset \Omega$ we have

$$
\lim _{k \rightarrow \infty} \int_{K}|\tilde{V}|\left|u_{k}\right|^{p} \mathrm{~d} x=0
$$


On the other hand, (8.5) and Hölder inequality imply that for any $\varepsilon>0$ there exists $K_{\varepsilon} \Subset \Omega$ such that

$$
\left.\left|\int_{\Omega \backslash K_{\varepsilon}}\right| \tilde{V}|| u_{k}\right|^{p} \mathrm{~d} x \mid \leq\left(\int_{\Omega \backslash K_{\varepsilon}}|\tilde{V}|^{d / p} W^{-d / p^{*}} \mathrm{~d} x\right)^{p / d}\left(\int_{\Omega} W\left|u_{k}\right|^{p^{*}} \mathrm{~d} x\right)^{p / p^{*}}<\varepsilon .
$$

Therefore, $\int_{\Omega}|\tilde{V}|\left|u_{k}\right|^{p} \mathrm{~d} x \rightarrow 0$. Since

$$
Q\left(u_{k}\right) \leq \tilde{Q}_{\lambda}\left(u_{k}\right)+|\lambda| \int_{\Omega}|\tilde{V}|\left|u_{k}\right|^{p} \mathrm{~d} x
$$

it follows that $Q\left(u_{k}\right) \rightarrow 0$. Hence, (8.4) implies that $\int_{\Omega} W\left|u_{k}\right|^{p^{*}} \mathrm{~d} x \rightarrow 0$, but this contradicts the assumption $\int_{\Omega} W\left|u_{k}\right|^{p^{*}} \mathrm{~d} x=1$. Consequently, (8.6) (resp. (8.7) ) holds true.

(iii) It follows from Proposition 8.3 that $S$ is an interval, and that $\lambda \in \operatorname{int} S$ implies that $\tilde{Q}_{\lambda}$ is subcritical in $\Omega$. The claim on the boundedness of $S$ is trivial and left to the reader.

On the other hand, suppose that for some $\lambda \in \mathbb{R}$ the functional $\tilde{Q}_{\lambda}$ is subcritical. By part (i), $\tilde{Q}_{\lambda}$ satisfies (8.6) with weight $W$. Therefore, (8.9) (with $K_{\varepsilon}=\emptyset$ ) implies that

$$
\tilde{Q}_{\lambda}(u) \geq C\left(\int_{\Omega} W|u|^{p^{*}} \mathrm{~d} x\right)^{p / p^{*}} \geq\left. C_{1}\left|\int_{\Omega} \tilde{V}\right| u\right|^{p} \mathrm{~d} x \mid \quad u \in C_{0}^{\infty}(\Omega) .
$$

Therefore, $\lambda \in \operatorname{int} S$. Consequently, $\lambda \in \partial S$ implies that $\tilde{Q}_{\lambda}$ is critical in $\Omega$. In particular, $0 \in$ int $S$.

Example 8.7. Let $2 \leq p<d$, and let $\Omega \subset \mathbb{R}^{d}$ be a bounded convex domain with a smooth boundary. Consider the Hardy functional

$$
Q(u):=\int_{\Omega}|\nabla u|^{p} \mathrm{~d} x-\left|\frac{p-1}{p}\right|^{p} \int_{\Omega} \frac{|u|^{p}}{d(x, \partial \Omega)^{p}} \mathrm{~d} x .
$$

By [11, the functional $Q$ satisfies the following Hardy-Sobolev-Maz'ya type inequality

$$
Q(u) \geq C\left(\int_{\Omega}|u|^{p^{*}} \mathrm{~d} x\right)^{p / p^{*}}
$$


Let $V \in L_{\text {loc }}^{\infty}(\Omega) \cap L^{d / p}(\Omega)$ be a positive function. By [19], there exists a constant $\lambda_{*}>0$ such that

$$
Q_{\lambda}(u):=Q(u)-\lambda \int_{\Omega} V|u|^{p} \mathrm{~d} x
$$

is nonnegative for all $\lambda \leq \lambda_{*}$. Now, Theorem 8.6 implies that the functional $Q_{\lambda_{*}}(u)$ is critical in $\Omega$. Moreover, for $\lambda<\lambda_{*}$ the following inequality holds

$$
Q_{\lambda}(u) \geq C_{\lambda}\left(\int_{\Omega}|u|^{p^{*}} \mathrm{~d} x\right)^{p / p^{*}} \quad u \in C_{0}^{\infty}(\Omega)
$$

for some $C_{\lambda}>0$.

Furthermore, for every nonzero nonnegative $\psi \in C_{0}^{\infty}(\Omega)$ the following inequality holds

$$
Q_{\lambda_{*}}(u)+C_{1}\left|\int_{\Omega} u \psi \mathrm{d} x\right|^{p} \geq C\left(\int_{\Omega}|u|^{p^{*}} \mathrm{~d} x\right)^{p / p^{*}} \quad u \in C_{0}^{\infty}(\Omega)
$$

for some $C, C_{1}>0$.

\section{The linear case $(p=2)$}

Some of the results in the preceding sections have stronger counterparts in the linear case (for a recent review on the theory of positive solutions of second-order linear elliptic PDEs, see [25] and the references therein). In particular, there are several properties that are true for the linear case but are generally false or unknown in the general case. This refers in particular to SCP whose scope of validity when $p \neq 2$ is not completely understood, and to the convexity of the functional $Q$, which is known to be generally false for $p>2$ (see references at the beginning of Section 7 ). On the other hand, as in [24, 27], we can actually consider in the linear symmetric case the following somewhat more general functional than $Q_{V}$ of the form (1.2).

Let $A: \Omega \rightarrow \mathbb{R}^{d^{2}}$ be a measurable symmetric matrix valued function such that for every compact set $K \Subset \Omega$ there exists $\mu_{K}>1$ so that

$$
\mu_{K}^{-1} I_{d} \leq A(x) \leq \mu_{K} I_{d} \quad \forall x \in K,
$$

where $I_{d}$ is the $d$-dimensional identity matrix, and the matrix inequality $A \leq B$ means that $B-A$ is a nonnegative matrix on $\mathbb{R}^{d}$. Let $V \in L_{\text {loc }}^{q}(\Omega)$ 
be a real potential, where $q>d / 2$. We consider the quadratic form

$$
\mathbf{a}_{A, V}[u]:=\frac{1}{2} \int_{\Omega}\left(A \nabla u \cdot \nabla u+V|u|^{2}\right) \mathrm{d} x
$$

on $C_{0}^{\infty}(\Omega)$ associated with the Schrödinger equation

$$
P u:=(-\nabla \cdot(A \nabla)+V) u=0 \quad \text { in } \Omega .
$$

We say that $\mathbf{a}_{A, V}$ is nonnegative on $C_{0}^{\infty}(\Omega)$, if $\mathbf{a}_{A, V}[u] \geq 0$ for all $u \in C_{0}^{\infty}(\Omega)$.

Let $v$ be a positive solution of the equation $P u=0$ in $\Omega$. Then by [27, Lemma 2.4] we have the following analog of (2.3). For any nonnegative $w \in C_{0}^{\infty}(\Omega)$ we have

$$
\mathbf{a}_{A, V}[v w]=\frac{1}{2} \int_{\Omega} v^{2} A \nabla w \cdot \nabla w \mathrm{~d} x .
$$

Moreover, it follows from [27, 28] that all the results mentioned in this paper concerning the functional $Q$ are also valid for the form $\mathbf{a}_{A, V}$.

\subsection{Liouville-type theorem}

In the linear case we have the following stronger Liouville-type statement (cf. Theorem 5.1 for $p \neq 2$ ).

Theorem 9.1 ([24]). Let $\Omega$ be a domain in $\mathbb{R}^{d}, d \geq 1$. Consider two strictly elliptic Schrödinger operators with real coefficients defined on $\Omega$ of the form

$$
P_{j}:=-\nabla \cdot\left(A_{j} \nabla\right)+V_{j} \quad j=0,1,
$$

where $V_{j} \in L_{\text {loc }}^{p}(\Omega)$ for some $p>d / 2$, and $A_{j}: \Omega \rightarrow \mathbb{R}^{d^{2}}$ are measurable symmetric matrices satisfying (9.1).

Assume that the following assumptions hold true.

(i) The operator $P_{1}$ admits a ground state $\varphi$ in $\Omega$.

(ii) $P_{0} \geq 0$ on $C_{0}^{\infty}(\Omega)$, and there exists a real function $\psi \in H_{\mathrm{loc}}^{1}(\Omega)$ such that $\psi_{+} \neq 0$, and $P_{0} \psi \leq 0$ in $\Omega$.

(iii) The following matrix inequality holds

$$
\left(\psi_{+}\right)^{2}(x) A_{0}(x) \leq C \varphi^{2}(x) A_{1}(x) \quad \text { a. e. in } \Omega,
$$

where $C>0$ is a positive constant. 
Then the operator $P_{0}$ is critical in $\Omega$, and $\psi$ is the corresponding ground state. In particular, $\psi$ is (up to a multiplicative constant) the unique positive supersolution of the equation $P_{0} u=0$ in $\Omega$.

\subsection{The space $\mathcal{D}_{A, V}^{1,2}$}

When $p=2$ and the quadratic form $\mathbf{a}_{A, V}$ defined by (9.2) is nonnegative, $\mathbf{a}_{A, V}$ induces a scalar product on $C_{0}^{\infty}(\Omega)$. One can regard as the natural domain of the functional

$$
\mathbf{a}_{A, V}^{f}(u):=\mathbf{a}_{A, V}[u]-\int_{\Omega} u f \mathrm{~d} x
$$

a linear space in which the functional $\mathbf{a}_{A, V}^{f}$ has a minimizer for all $f$ such that $\mathbf{a}_{A, V}^{f}$ is bounded from below. The functional $\mathbf{a}_{A, V}^{f}$ is bounded from below if and only if $\int_{\Omega} u f \mathrm{~d} x$ is a continuous functional with respect to the norm $\left(\mathbf{a}_{A, V}[u]\right)^{1 / 2}$. The minimum for such $f$ is not attained on $C_{0}^{\infty}(\Omega)$ due to the strong maximum principle, but any minimizing sequence for $\mathbf{a}_{A, V}^{f}$ is a Cauchy sequence. Thus, the natural domain of $\mathbf{a}_{A, V}$ is the completion of $C_{0}^{\infty}(\Omega)$ in the norm $\left(\mathbf{a}_{A, V}[u]\right)^{1 / 2}$. In the subcritical case, due to (3.4) (which is valid also for subcritical operators of the form (9.3)), this completion is continuously imbedded into $W^{1,2}(\Omega ; W)$ for some positive continuous function $W$. By analogy with the classical space $\mathcal{D}^{1,2}$, we denote the completion space with respect to the above norm in the subcritical case by $\mathcal{D}_{A, V}^{1,2}(\Omega)$ (see [27]).

If, however, $\mathbf{a}_{A, V}$ has a ground state $v$, the span of $v$ becomes obviously the zero element of the completion space with respect to the norm $\left(\mathbf{a}_{A, V}[u]\right)^{1 / 2}$. Recalling the definition of $\mathcal{D}^{1,2}\left(\mathbb{R}^{d}\right)$ for $d=1,2$, where the ground state of $\mathbf{a}_{I, 0}[u]=\int_{\mathbb{R}^{d}}|\nabla u|^{2} \mathrm{~d} x$ is $\mathbf{1}$, and in light of (3.2), we define in the critical case the norm

$$
\|u\|:=\left(\mathbf{a}_{A, V}[u]+\left|\int_{\Omega} \psi u \mathrm{~d} x\right|^{2}\right)^{1 / 2},
$$

where $\psi$ is any $C_{0}^{\infty}(\Omega)$-function satisfying $\int_{\Omega} \psi v \mathrm{~d} x \neq 0$. Hence, also in the critical case the completion of $C_{0}^{\infty}(\Omega)$ with respect to the norm defined by (9.7) (which we also denote by $\mathcal{D}_{A, V}^{1,2}(\Omega)$ ) is continuously imbedded into the function space $W^{1,2}(\Omega ; W)$, with an appropriate weight function $W$. 
Example 9.2. Let $\Omega=\mathbb{R}^{d}=\mathbb{R}^{n} \times\left(\mathbb{R}^{m} \backslash\{0\}\right), 1 \leq m \leq d$, and denote points in $\Omega$ by $(x, y) \in \mathbb{R}^{n} \times\left(\mathbb{R}^{m} \backslash\{0\}\right)$. Let

$$
\mathbf{a}[u]=\frac{1}{2} \int_{\Omega}|\nabla u|^{2} \mathrm{~d} x \mathrm{~d} y-\left(\frac{m-2}{2}\right)^{2} \int_{\Omega} \frac{u^{2}}{|y|^{2}} \mathrm{~d} x \mathrm{~d} y \quad u \in C_{0}^{\infty}(\Omega) .
$$

The functional (9.8) is nonnegative due to the Hardy inequality and the constant $\left(\frac{m-2}{2}\right)^{2}$ is the maximal constant for which this is true. Furthermore, if $m<d$, the functional (9.8) is weakly coercive, while for $m=d$ it has a generalized ground state $v(y)=|y|^{(2-m) / 2}$.

It follows that completion of $C_{0}^{\infty}(\Omega)$ in the norm induced by (9.8) for $m<d$ defines a natural domain for the functional (9.8). It should be noted, however, that on the complete space the integrals in the expression (9.8) might be infinite. A more explicit characterization of the natural domain in this case can be obtained by noting that $v(x, y)=|y|^{(2-m) / 2}$ is a positive solution of the corresponding equation. Thus, for the functional (9.8) the positive Lagrangian identity (2.1) gives

$$
\mathbf{a}[u]=\frac{1}{2} \int_{\Omega}|y|^{2-m}\left|\nabla\left(|y|^{(m-2) / 2} u\right)\right|^{2} \mathrm{~d} x \mathrm{~d} y .
$$

It follows that the completion space can be characterized as a space of measurable functions with measurable weak derivatives for which the integral in (9.9) is finite.

An analogous definition of the natural domain for the functional $Q_{V}$ could be given for general $p$ whenever the functional $Q_{V}$ is convex. We should point out that while this is in general false, one may require the convexity of another functional $\hat{Q}$, bounded by $Q_{V}$ from above and from below. In particular, one can look at the functionals (2.5) or (2.6). The following statement from [30]) characterizes a convexity property of $\hat{Q}$ given by the right hand side of (2.6) .

Proposition 9.3. Let $p>2$, and let $v \in C_{\mathrm{loc}}^{1}(\Omega)$ be a fixed positive function. Then the functional

$$
\mathcal{Q}(u):=\int_{\Omega}\left[v^{p}\left|\nabla\left(u^{2 / p}\right)\right|^{p}+v^{2}|\nabla v|^{p-2} u^{2(p-2) / p}\left|\nabla\left(u^{2 / p}\right)\right|^{2}\right] \mathrm{d} x
$$

is convex on $\left\{u \in C_{0}^{\infty}(\Omega), u \geq 0\right\}$. 


\subsection{Positive solutions of minimal growth}

We characterize now positive solutions of minimal growth in a neighborhood of infinity of $\Omega$ in terms of a modified null sequence of the form $\mathbf{a}_{A, V}$ (cf. Section 6).

Theorem 9.4 ([29, Theorem 6.1]). Suppose that $\mathbf{a}_{A, V}$ is nonnegative on $C_{0}^{\infty}(\Omega)$. Let $\Omega_{1} \Subset \Omega$ be an open set, and let $u \in C\left(\Omega \backslash \Omega_{1}\right)$ be a positive solution of the equation $P u=0$ in $\Omega \backslash \overline{\Omega_{1}}$.

Then $u \in \mathcal{M}_{\Omega, \overline{\Omega_{1}}}$ if and only if for every smooth open set $\Omega_{2}$ satisfying $\Omega_{1} \Subset \Omega_{2} \Subset \Omega$, and an open set $B \Subset\left(\Omega \backslash \overline{\Omega_{2}}\right)$ there exists a sequence $\left\{u_{k}\right\} \subset$ $C_{0}^{\infty}(\Omega), u_{k} \geq 0$, such that for all $k \in \mathbb{N}, \int_{B}\left|u_{k}\right|^{2} \mathrm{~d} x=1$, and

$$
\lim _{k \rightarrow \infty} \int_{\Omega \backslash \bar{\Omega}_{2}} u^{2} A \nabla u_{k} \cdot \nabla u_{k} \mathrm{~d} x=0 .
$$

Consider now the following Phragmén-Lindelöf-type principle that holds in unbounded or nonsmooth domains, and for irregular potential $V$, provided the subsolution satisfies a certain decay property (of variational type) in terms of the Lagrangian $L$ (cf. [1, 15, 18, 31] and Section [6).

Theorem 9.5 (Comparison Principle [29]). Assume that $P$ is a nonnegative Schrödinger operator of the form (9.3). Fix smooth open sets $\Omega_{1} \Subset \Omega_{2} \Subset \Omega$. Let $u, v \in W_{\text {loc }}^{1, p}\left(\Omega \backslash \Omega_{1}\right) \cap C\left(\Omega \backslash \Omega_{1}\right)$ be, respectively, a positive subsolution and a supersolution of the equation $P w=0$ in $\Omega \backslash \overline{\Omega_{1}}$ such that $u \leq v$ on $\partial \Omega_{2}$.

Assume further that $P u \in L_{\mathrm{loc}}^{\infty}\left(\Omega \backslash \Omega_{1}\right)$, and that there exist an open set $B \Subset\left(\Omega \backslash \overline{\Omega_{2}}\right)$ and a sequence $\left\{u_{k}\right\} \subset C_{0}^{\infty}(\Omega), u_{k} \geq 0$, such that

$$
\int_{B}\left|u_{k}\right|^{p} \mathrm{~d} x=1 \quad \forall k \geq 1, \text { and } \quad \lim _{k \rightarrow \infty} \int_{\Omega \backslash \bar{\Omega}_{1}} u^{2} A \nabla\left(u_{k} / u\right) \cdot \nabla\left(u_{k} / u\right) \mathrm{d} x=0 .
$$

Then $u \leq v$ on $\Omega \backslash \Omega_{2}$.

Remark 9.6. In Theorem 9.5 we assumed that the subsolution $u$ is strictly positive. It would be useful to prove the above comparison principle under the assumption that $u \geq 0$ (cf. [15]). 


\section{Acknowledgments}

Y. P. acknowledges the support of the Israel Science Foundation (grant No. 587/07) founded by the Israeli Academy of Sciences and Humanities, by the Fund for the Promotion of Research at the Technion, and by the Technion President's Research Fund.

\section{References}

[1] S. Agmon, Bounds on exponential decay of eigenfunctions of Schrödinger operators, in "Schrödinger Operators" (Como, 1984), pp. 1-38, Lecture Notes in Math. 1159, Springer, Berlin, 1985.

[2] G. Alessandrini, and M. Sigalotti, Geometric properties of solutions to the anisotropic $p$-Laplace equation in dimension two, Ann. Acad. Sci. Fenn. Math. 26 (2001), 249-266.

[3] W. Allegretto, and Y. X. Huang, A Picone's identity for the $p$-Laplacian and applications, Nonlinear Anal. 32 (1998), 819-830.

[4] W. Allegretto, and Y. X. Huang, Principal eigenvalues and Sturm comparison via Picone's identity, J. Differential Equations 156 (1999), 427438.

[5] M. Cuesta, and P. Takáč, A strong comparison principle for positive solutions of degenerate elliptic equations, Differential Integral Equations 13 (2000), 721-746.

[6] J. I. Diaz, and J. E. Saá, Existence et unicité de solutions positives pour certaines équations elliptiques quasilinéaires, C. R. Acad. Sci. Paris Ser. I Math. 305 (1987), 521-524.

[7] L. Damascelli, and B. Sciunzi, Harnack inequalities, maximum and comparison principles, and regularity of positive solutions of $m$-Laplace equations, Calc. Var. Partial Differential Equations 25 (2006), 139-159.

[8] M. del Pino, M. Elgueta, and R. Manasevich, A homotopic deformation along $p$ of a Leray-Schauder degree result and existence for $\left(\left|u^{\prime}\right|^{p-2} u^{\prime}\right)^{\prime}+$ $f(t, u)=0, u(0)=u(T)=0, p>1$ J. Differential Equations 80 (1989), 1-13. 
[9] P. Drábek, A. Kufner, and F. Nicolosi, "Quasilinear Elliptic Equations with Degenerations and Singularities", de Gruyter Series in Nonlinear Analysis and Applications 5, Walter de Gruyter \& Co., Berlin, 1997.

[10] I. Ekeland, and R. Temam, "Convex Analysis and Variational Problems", North Holland-American Elsevier, Amsterdam, 1976.

[11] S. Filippas, V. Maz'ya, and A. Tertikas, Critical Hardy-Sobolev inequalities. J. Math. Pures Appl. (9) 87 (2007), 37-56.

[12] J. Fleckinger-Pellé, J. Hernández, P. Takáč, and F. de Thélin, Uniqueness and positivity for solutions of equations with the $p$-Laplacian, Proceedings of the Conference on Reaction-Diffusion Equations (Trieste, 1995), pp. 141-155, Lecture Notes in Pure and Applied Math. Vol. 194, Marcel Dekker, New York, 1998.

[13] J. García-Melián, and J. Sabina de Lis, Maximum and comparison principles for operators involving the $p$-Laplacian, J. Math. Anal. Appl. 218 (1998), 49-65.

[14] O. A. Ladyženskaja, and N. N. Ural'ceva, A variational problem for quasilinear elliptic equations with many independent variables, Soviet Math. Dokl. 1 (1960), 1390-1394.

[15] V. Liskevich, S. Lyakhova, and V. Moroz, Positive solutions to nonlinear $p$-Laplace equations with Hardy potential in exterior domains, $J$. Differential Equations 232 (2007), 212-252.

[16] M. Lucia, and S. Prashanth, Strong comparison principle for solutions of quasilinear equations, Proc. Amer. Math. Soc. 132 (2004), 1005-1011.

[17] J. J. Manfredi, Isolated singularities of $p$-harmonic functions in the plane, SIAM J. Math. Anal. 22 (1991), 424-439.

[18] M. Marcus, V. J. Mizel, and Y. Pinchover, On the best constant for Hardy's inequality in $\mathbb{R}^{n}$, Trans. Amer. Math. Soc. 350 (1998), 32373255 .

[19] M. Marcus, and I. Shafrir, An eigenvalue problem related to Hardy's $L^{p}$ inequality, Ann. Scuola Norm. Sup. Pisa Cl. Sci. (4) 29 (2000), 581-604. 
[20] Mitidieri, and S. I. Pokhozhaev, Some generalizations of Bernstein's theorem, Differ. Uravn. 38 (2002), 373-378; translation in Differ. Equ. 38 (2002), 392-397.

[21] M. Murata, Structure of positive solutions to $(-\Delta+V) u=0$ in $\mathbb{R}^{n}$, Duke Math. J. 53 (1986), 869-943.

[22] Y. Pinchover, On positive solutions of second-order elliptic equations, stability results, and classification, Duke Math. J. 57 (1988), 955-980.

[23] Y. Pinchover, On criticality and ground states of second order elliptic equations, II, J. Differential Equations 87 (1990), 353-364.

[24] Y. Pinchover, A Liouville-type theorem for Schrödinger operators, Comm. Math. Phys. 272 (2007) 75-84.

[25] Y. Pinchover, Topics in the theory of positive solutions of second-order elliptic and parabolic partial differential equations, to appear in "Spectral Theory and Mathematical Physics: A Festschrift in Honor of Barry Simon's 60th Birthday", Proceedings of Symposia in Pure Mathematics 76, American Mathematical Society, Providence, RI, 2007, 329-356.

[26] Y. Pinchover, A. Tertikas, and K. Tintarev, A Liouville-type theorem for the $p$-Laplacian with potential term, Ann. Inst. H. Poincaré-Anal. Non Linéaire 25 (2008), 357-368.

[27] Y. Pinchover, and K. Tintarev, Ground state alternative for singular Schrödinger operators, J. Functional Analysis, 230 (2006), 65-77.

[28] Y. Pinchover, and K. Tintarev, Ground state alternative for $p$-Laplacian with potential term, Calc. Var. Partial Differential Equations 28 (2007), 179-201.

[29] Y. Pinchover, and K. Tintarev, On positive solutions of minimal growth for singular $p$-Laplacian with potential term, Adv. Nonlinear Stud. 8 (2008), 213-234.

[30] Y. Pinchover, and K. Tintarev, On the Hardy-Sobolev-Maz'ya inequality and its generalizations, in "Sobolev Spaces in Mathematics I: Sobolev Type Inequalities", ed. V. Maz'ya, International Mathematical Series 8, Springer, 2009, 281-297. 
[31] A. Poliakovsky, and I. Shafrir, Uniqueness of positive solutions for singular problems involving the $p$-Laplacian, Proc. Amer. Math. Soc. 133 (2005), 2549-2557.

[32] P. Pucci, and J. Serrin, "The Maximum Principle", Progress in Nonlinear Differential Equations and their Applications, 73. Birkhäuser Verlag, Basel, 2007.

[33] W. Rudin, "Functional Analysis", 2nd ed., McGraw-Hill, Inc., New York, 1991.

[34] J. Serrin, Local behavior of solutions of quasi-linear equations, Acta Math. 111 (1964), 247-302.

[35] J. Serrin, Isolated singularities of solutions of quasi-linear equations, Acta Math. 113 (1965), 219-240.

[36] P. Takáč, and K. Tintarev, Generalized minimizer solutions for equations with the $p$-Laplacian and a potential term, Proc. Roy. Soc. Edinburgh Sect. A 138 (2008), 201-221.

[37] P. Tolksdorf, On the Dirichlet problem for quasilinear equations in domains with conical boundary points, Comm. Partial Differential Equations 8 (1983), 773-817.

[38] P. Tolksdorf, Regularity for a more general class of quasilinear elliptic equations, J. Differential Equations 51 (1984), 126-150.

[39] M. Troyanov, Parabolicity of manifolds, Siberian Adv. Math. 9 (1999), $125-150$.

[40] M. Troyanov, Solving the $p$-Laplacian on manifolds, Proc. Amer. Math. Soc. 128 (2000), 541-545. 